\title{
NOTAS DE ANÁLISE COMBINATÓRIA NA MATEMÁTICA ISLÂMICA
}

\author{
COMBINATORY ANAL YSIS NOTES IN ISLAMIC MATHEMATICS
}

\author{
Davidson Paulo Azevedo Oliveira ${ }^{1}$
}

\begin{abstract}
RESUMO
Estudos apontam para uma escassez de pesquisas sobre a História da Matemática Islâmica em Língua Portuguesa, não sendo diferente se olharmos para o conteúdo de Análise combinatória. Quando, no Brasil, é retratado o raciocínio combinatório, a discussão normalmente fica restrita à Europa. Nesse sentido, o objetivo deste relato é apresentar notas históricas do pensamento combinatório não eurocêntricas, especificamente, islâmicas. Para isto, fizemos um estudo bibliográfico baseado em fontes secundárias dos principais pesquisadores internacionais da área. Destacamos que eles seguem uma linha historiográfica tradicional ao olhar para os textos originais com olhos do presente. São apresentadas neste estudo notas sobre três estudiosos islâmicos de períodos distintos, mas que estão, de certo modo, conectados uns aos outros. O primeiro deles, Ibn al-Banna (1256 - 1321), que viveu em Maraquexe, afirma em sua obra que se baseou no trabalho de al-Muncin (? - 1228), também dessa cidade. Esse é o segundo estudioso a quem dedicamos alguns parágrafos para comentar sobre a seção XI de seu principal manuscrito, na qual descrevia métodos e problemas de Análise combinatória, sendo uma ampliação do trabalho de alKhalil (718 - 798), linguista e lexicógrafo que viveu em Baçorá, atual Iraque. Além de ser considerado o autor do primeiro dicionário de Língua Árabe, al-Khalil fez uso do pensamento combinatório para calcular o número de palavras que se poderia escrever com até cinco letras. Por meio dessas notas históricas podemos perceber como a Análise combinatória foi utilizada por estudiosos de matemática e linguistas no medievo islâmico, reunindo informações úteis a professores e pesquisadores. Esperamos que o caráter não eurocêntrico, especialmente no que diz respeito à perspectiva islâmica, possa ter sido levantado nessas curtas notas históricas. Ressaltamos a possibilidade e a necessidade de pesquisas mais profundas sobre o tema em Língua Portuguesa e baseadas em textos originais.
\end{abstract}

Palavras-chave: Análise combinatória; Matemática Islâmica; Não-eurocentrismo.

\begin{abstract}
Studies indicate a lack of research on the History of Islamic Mathematics in Portuguese, which is no different if we consider the content of Combinatorial Analysis. When the History of Mathematics is discussed in Brazil, it is usually restricted to Europe. In this sense, the purpose of this work is to present historical notes on non-Eurocentric combinatorial thinking, specifically in regard to Islamic Civilization. In order to do so, we carried out a bibliographic study based on secondary sources from the main international researchers in this field. We emphasize that they

1 Doutor em Educação Matemática pela Universidade Estadual Paulista (UNESP). Professor do Departamento de Matemática do Centro Federal de Educação Tecnológica de Minas Gerais (CEFET-MG), Belo Horizonte, Minas Gerais, Brasil. Avenida Amazonas, 7675, Departamento de Matemática, Nova Gameleira, Belo Horizonte, Minas Gerais, CEP: 30510 - 000. E-mail: davidson@ cefetmg.br
\end{abstract}

(iD) ORCID iD: https://orcid.org/0000-0003-2794-8515 
follow a traditional line of historiography, approaching the original texts from a contemporary perspective. In this study, we present notes on three Islamic scholars from different periods, who are, however, related to each other. The first of them, Ibn al-Banna (1256 - 1321), lived in Marakesh and stated that his work was based on that of al-Muncin (? - 1228), from the same city. Al-Mucin is the second scholar to whom we have dedicated a few paragraphs. We comment on section XI of his main manuscript, in which he described methods and problems of Combinatorial Analysis in an extension of the work of al-Khalil (718 - 798) - a linguist and lexicographer who lived in Basra, nowadays Iraq. Al-Khalil, the third scholar presented in this study, is considered to be the author of the first Arabic Language dictionary and used combinatorial thinking to calculate the number of words that could be written with up to five letters. Through these historical notes, we can understand how Combinatorial Analysis was used by mathematical scholars and linguists in the Islamic civilization in Middle Ages and we can assemble useful information for teachers and researchers of mathematics. By focusing on the Islamic understanding of Combinatorial Analysis, we hope to raise a non-Eurocentric perspective with these short historical notes. We also point out the need for further studies on this topic, based on original texts and that can be published in Portuguese.

Keywords: Combinatorial Analysis; Islamic Mathematics; Non-Eurocentrism. 


\section{Introdução}

Enquanto professor de Matemática do Ensino Médio ministro aulas de diversos conteúdos, dentre eles, Análise combinatória em turmas de Segunda Série. Para preparar as aulas sobre permutações, arranjos, combinações considero importante o hábito de me inteirar das pesquisas em Educação Matemática que discutem o tema. Nessa busca por materiais diversos me interessa sempre a História da Matemática e como ela pode ser utilizada pelo professor em sala de aula.

Além das tarefas didáticas dedico-me, desde o início de 2020, ao estudo sobre a História da Matemática no mundo Islâmico Medieval que, conforme afirmam Morey, Oliveira e Pereira (2021), é uma das civilizações que carecem de relatos historiográficos. De fato, ao olhar para as pesquisas e literatura no Brasil sobre Análise combinatória (BASTOS, 2020; MARTINS e BONFIM, 2011) podemos ressaltar o caráter eurocêntrico que assumem ao serem quase que na totalidade restritas à Europa do século XVII.

Ao tratar do histórico da Análise combinatória em um contexto geográfico não eurocêntrico podemos perceber uma prevalência de trabalhos que se restringem aos quadrados mágicos na matemática chinesa e islâmica, ou a famosa poesia de Santi Yves que perpassou diversas culturas com distintos versos, inclusive no Papiro de Rhind. Esses dois exemplos são apresentados, por exemplo, no minicurso ministrado por Vasquez e Noguti (2004) no VIII Encontro Nacional de Educação Matemática no qual as autoras propõem problemas de Análise combinatória baseados em estudiosos europeus.

A partir de outro minicurso ministrado no Seminário Nacional de História da Matemática, originou-se o trabalho de Martins e Bonfim (2011) com questões mais aprofundadas sobre aspectos históricos de Análise combinatória. As autoras mencionam o mundo islâmico medieval em alguns parágrafos e afirmam que suas contribuições mais significativas foram para os quadrados mágicos e números binomiais, sem discorrer muito a respeito.

Em um estudo mais recente podemos apresentar a dissertação de Mestrado de Bastos (2020) que investigou modelos envolvendo o pensamento combinatório e solicitou aos alunos e alunas participantes da investigação, que realizassem uma pesquisa sobre História da Análise combinatória. Os resultados apresentados por eles priorizam resultados eurocêntricos com Pascal, Leibniz, entre outros europeus. O próprio autor no 
início de seu trabalho não menciona a civilização islâmica e suas contribuições para o estudo de combinações, permutações e arranjos.

Diante deste contexto de escassez de materiais que tratem de Análise combinatória no medievo islâmico para além de quadrados mágicos e triângulos aritméticos, apresentaremos algumas notas sobre o assunto ao tratarmos sobre três estudiosos islâmicos e seus trabalhos em análise combinatória. Ressaltamos que, por se tratar de considerações iniciais sobre o tema não há um aprofundamento das questões contextuais envolvidas, nem na matemática, nem nos problemas por eles resolvidos.

Berggren (2016) enuncia nomes de islâmicos que apresentaram contribuições ligadas à análise combinatória. Ele inicia com o alquimista Jābir b. Hayyān (721 - 813) de Kufa, Iraque; em seguida ressalta Thabit ibn Qura (836 - 910) que enumerou os modos como os raios aparecem no Teorema de Menelaus e, por fim cita al - Biruni (973 - 1048) que enumerou todos os casos possíveis de triângulos esféricos.

Entretanto, Berggren (2016) destaca que apesar dessas contribuições uma teoria para o cálculo combinatório aparece pela primeira vez em Magrebe e em Andaluz, especificamente com al-Muncin (? - 1228),. Nesse sentido, trataremos nesse trabalho de al-Muncim bem como de uma influência anterior a ele, o linguista al-Khalil (718 - 798), e de uma outra figura posterior a al-Muncim, o matemático e astrônomo al-Banna (12561321), cujo trabalho foi por este influenciado.

A partir dos três podemos perceber, como destaca Djebbar (1985), as influências e interesses de estudiosos islâmicos de Magrebe que se ocuparam de combinatória. Segundo ele, os islâmicos são provenientes de duas tradições:

a. linguística e gramática árabes e, também atividades astrológicas.

b. astronomia, música e álgebra (sendo essa uma tendência mais forte).

Nosso objetivo é apresentar notas históricas de Análise combinatória em perspectiva não eurocêntrica, especificamente islâmica. É um estudo bibliográfico no qual não recorremos a fontes originais, mas utilizamos fontes secundárias que são, de acordo com Pontes, Batista e Pereira (2021, p. 4) "aquelas que já passaram por algum tratamento, ou interpretação por parte de outros e que foram escritas sob a concepção deles". Ressaltamos, também, que os principais autores aqui apresentados (DARWISH, 1955; DJEBBAR, 1985; ABALLAGH, 1988; RASHED, 1994; ABDELJAOUAD, 2002, 
Davidson Paulo Azevedo Oliveira

Notas de Análise Combinatória na Matemática Islâmica

BERGGREN, 2016;) seguem uma linha historiográfica tradicional ao olhar para os textos originais com um olhar do presente.

Nosso texto está estruturado não a partir de uma ordem cronológica, mas a estrutura perfaz o caminho que trilhamos em nosso estudo: a primeira seção apresenta um primeiro contato com al-Banna que deixou pistas que levaram a al-Muncim, sendo a este dedicada a segunda seção deste texto. Em seguida, a terceira seção segue as pistas deixadas por al-Muncim que citou os trabalhos de al-Khalil, a quem está dedicada esta parte. Por fim, encerramos o texto com algumas reflexões sobre a Análise combinatória em civilizações não europeias.

\section{Ibn al-Banna}

Abu al- ${ }^{\mathrm{C}}$ Abbas Ahmab ibn Muhammad al-Azdi al-Murrakushi, ou como mais conhecido, Ibn al-Banna é originário de Marraquexe onde viveu e morreu. Ele ia constantemente a Fez a convite do rei em razão de seu conhecimento em astrologia. Além de astrologia, ele estudou matemática, o Alcorão, a língua árabe, a prosódia, a ciência das heranças, o Hadith, a sunna, medicina e astronomia (ABALLAGH, 1988).

Dentre seus escritos matemáticos podemos citar Kitab Talkhis $A^{c}$ mal al-Hisab que versava sobre cálculos, sendo esse texto o mais conhecido pelos historiadores do século XX devido a uma tradução francesa do mesmo. A partir de Talkhis, ele escreve um comentário, Raf c al-Hijab, que é um tratado de aritmética e álgebra no qual ele explicita como utiliza análise combinatória. De acordo com Aballagh (1988) esta obra é a que mais permite a historiadores analisarem as contribuições matemáticas de al-Banna e sua originalidade, especialmente em álgebra e teoria dos números. Ele relaciona cálculo combinatório com teoria de números como se segue:

\footnotetext{
A soma dos quadrados é útil para a composição de palavras de três letras para enumerar o conteúdo de idiomas e de coisas parecidas, por exemplo: Quantas palavras de três letras temos a partir das letras do alfabeto de acordo com uma figura sem permutação? De fato, o número de palavras de três letras e igual a soma dos números triangulares dados que o lado do último é inferior ao número dessas letras do alfabeto de duas letras e a soma dos números triangulares se obtém ao multiplicar o lado do último pelo produto dos dois números inteiros, que o seguem, e tomados o seis como resultado, como que fazemos a soma dos quadrados ímpares e dos quadrados pares² (ABALLAGH, 1988, p. 65-66, tradução nossa).
}

\footnotetext{
${ }^{2}$ La sommation des carrés est utile dans la composition des mot trilitéres pour dénombrer [le contenu] des langues et de [choses] semblables par exemple: Combien [a-t-on] de mots trilitéres à partir des lettres de
} 
Além disso, em um dos problemas ele calcula o número de combinações de $n$ elementos tomados $\mathrm{p}$ a $\mathrm{p}$ sem o uso de tabelas, por meio de raciocínio aritmético e recursivo. Estuda, ainda, as propriedades de números poligonais e estabelece relação entre eles. O raciocínio recursivo pode ser visto ao comparar os números poligonais com o número de combinações possíveis de palavras ao se combinar as letras do alfabeto árabe.

\footnotetext{
Se temos dadas vinte e oito letras, quantas palavras de cinco letras podemos formar? Faremos como se sucede: o resultado será, como no exemplo, o produto de cinco por vinte e quatro, multiplicado por treze e depois por nove e por sete ${ }^{3}$ (ABALLAGH, 1988, p. 69, tradução nossa).
}

Em outro manuscrito Raising the Veil Ibn al-Banna discute a solução de problemas parecidos sobre como encontrar a quantidade de palavras que se podem formar escolhendo-se um número de letras de uma quantidade definida de letras dadas (BERGGREN, 2016). Além disso, em outro texto ele comenta que está se baseando no trabalho de al-Muncim, conforme defende Djebbar (1985, p.4, tradução nossa): "Quanto ao número de palavras de três ou quatro letras ou então o número de letras é diferente, mas constituído a partir das vinte e oito letras do alfabeto árabe, Ibn Muncim construiu para isso uma tabela ${ }^{4}$ ".

De acordo com Abdeljaouad (2002), al-Banna foi responsável por popularizar o trabalho e as ideias de al-Muncim, portanto, merece também que dediquemos algumas notas ao manuscrito Fiqh al-Hisab. A ligação entre al-Banna e al-Muncim provavelmente se deu por Qadi ash-Sharif que foi aluno de al-Muncim e professor de al-Banna.

\section{Al-Muncim}

Al-Muncim é um árabe que nasceu em Andaluz e não há indícios de sua data de nascimento. Sabe-se que ele vai para Marraquexe onde morre em 1228. Uma das poucas informações que, até então, se tem é a de que al-Banna teve acesso ao seu manuscrito, o

\footnotetext{
l'alphabet selon une seule figure, sans ses permutées? En effet, [le nombre] des mots trilitéres est égal à la somme des trigones dont le côté du dernier est inférieur au nombre [de ces lettres de l'alphabet] de deux; et la somme des trigones s'obtient en multiplicant le côté du dernier par le produit des deux nombres [entiers], qui le suivent, et en prenant le sixième du résultat, comme ce que l'on fait pour la sommation des carrés des impairs, et des carrés des pairs.

${ }^{3} \mathrm{Si}$ on dit [étant donné] vingt huit lettres combien de mots quintilitères [peut-on] en composer? Procèdes selon ce qui précede le résultat sera, dans cet exemple: le produit de cinq par vingt quatre, multiplié par treize, puis par neuf, puis par sept.

${ }^{4}$ Quant au nombre de mots trilitères ou quadrilitères ou dont le nombre de lettres est autre et qui sont constitués à partir des vingt huit lettres de l'alphabet, Ibn Muncim a dressé pour cela un tableau.
} 
qual serviu de base para seu próprio desenvolvimento em análise combinatória. Al-Abili, ex aluno de al-Banna, também cita al-Muncim em seus manuscritos. Por esse motivo, Djebbar (1985) afirma que al-Muncim foi conhecido no século XIII e XIV no mundo muçulmano ocidental.

Ele escreve uma obra denominada Du Fiqh al-Hisab, que em uma tradução livre pode ser entendida como A Jurisprudência da Aritmética, a qual é um manuscrito voltado a estudantes. De acordo com Djebbar (1985), foi escrito, provavelmente, entre 1207 e 1212, em Marraquexe. Berggren (2016) também concorda que ele tenha sido escrito no século XIII e o classifica como um dos mais importantes manuscritos de análise combinatória do período.

Essa obra está dividida em duas partes e, de acordo com Djebbar (1985) tinha o objetivo de apresentar em um mesmo manuscrito livros teóricos e práticos, algo que não existia na época. Al-Muncim divide esse trabalho em duas partes: sendo onze seções na primeira e seis na segunda. A seção que nos interessa é a XI da primeira parte, e é classificada pelo historiador Djebbar (1985) como sendo de aspectos teóricos. Além disso, o pesquisador ressalta a importância que esse manuscrito possui por representar uma mudança de olhar em relação à análise combinatória que, até então, era discutida por meio de tabelas.

Al-Muncim não ignora completamente as tabelas, mas apresenta explicações de raciocínios que podem ser realizados para a generalização dos resultados. Como o texto é destinado a estudantes, ele continua apresentando tabelas, num total de dez.

No trabalho al-Muncim apresenta todos as possíveis palavras escritas em árabe que podem ser formadas de até dez letras considerando as 28 do alfabeto. Berggren (2016) destaca que o idioma tem algumas particularidades e compara-as com algumas da Língua Inglesa. Cita uma frase de al-Muncim na qual ele ilustra que as particularidades da língua árabe e a restrição de 28 letras possíveis é um detalhe, sendo que esse mesmo raciocínio pode ser utilizado para todo e qualquer problema.

No entanto, para se chegar a este problema o Fiqh al-Hisab é dividido em dez problemas iniciando com uma questão que, inicialmente, não tem relação com o objetivo principal de encontrar o número de palavras que se pode formar a partir de uma determinada quantidade de letras. O primeiro problema é mais prático e trata de um 
Davidson Paulo Azevedo Oliveira

Notas de Análise Combinatória na Matemática Islâmica

raciocínio mais tridimencional. Djebbar (1985) traduziu o manuscrito para o francês e apresentamos abaixo uma tradução livre para o português deste primeiro problema:

Dadas dez cores de seda, com as quais queremos fazer borlas de uma cor, duas cores, três cores, e assim sucessivamente até que a última borla que é dada pelas dez cores de seda seja construída. Queremos saber qual é o número de borlas de cada tipo, dada que as cores de cada borla são conhecidas e qual o número de todas as borlas juntas tendo em vista as diferentes cores de borlas ${ }^{5}$ (DJEBBAR, 1985, p. 50, tradução nossa).

O problema é resolvido por meio de um raciocínio combinatório e por meio de recorrência, o resultado de três cores dependendo do de duas cores. Ao final, al-Muncim apresenta a resolução por meio de uma tabela (figura 1) e ressalta que é possível o leitor observar resultados interessantes. Ele não aprofunda quais são os resultados, mas deixa pistas de que conhecia as propriedades do triângulo aritmético. Após esse comentário, AlMuncim retoma a tabela e explica como poderia ser realizada a resolução por meio da consulta e construção do triângulo aritmético.

\footnotetext{
${ }^{5}$ Etant donné dix coulerus de soie, avec lesquelles nous voulons faire des houppes [respectivement] d'une, de deux, de trois coulerus et ainsi de suite, jusqu'à la dernière houppe qui doit être de dix couleurs, nous voulons savoir quel est le nombre de houppes de chaque espèce, les couleurs de chaque houppe étant connues, ou quel est le nombre de toutes les houppes rassemblées, compte tenu des diffe'rentes nombres de coulerus des houppes.
} 
Figura 1 - Tabela de Resolução do Primeiro Problema

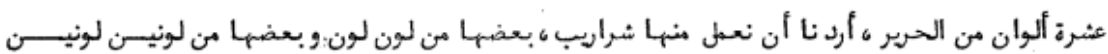

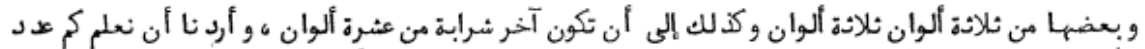

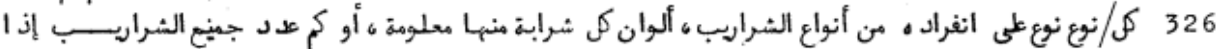

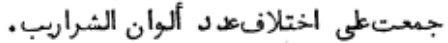

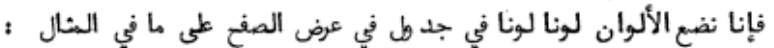

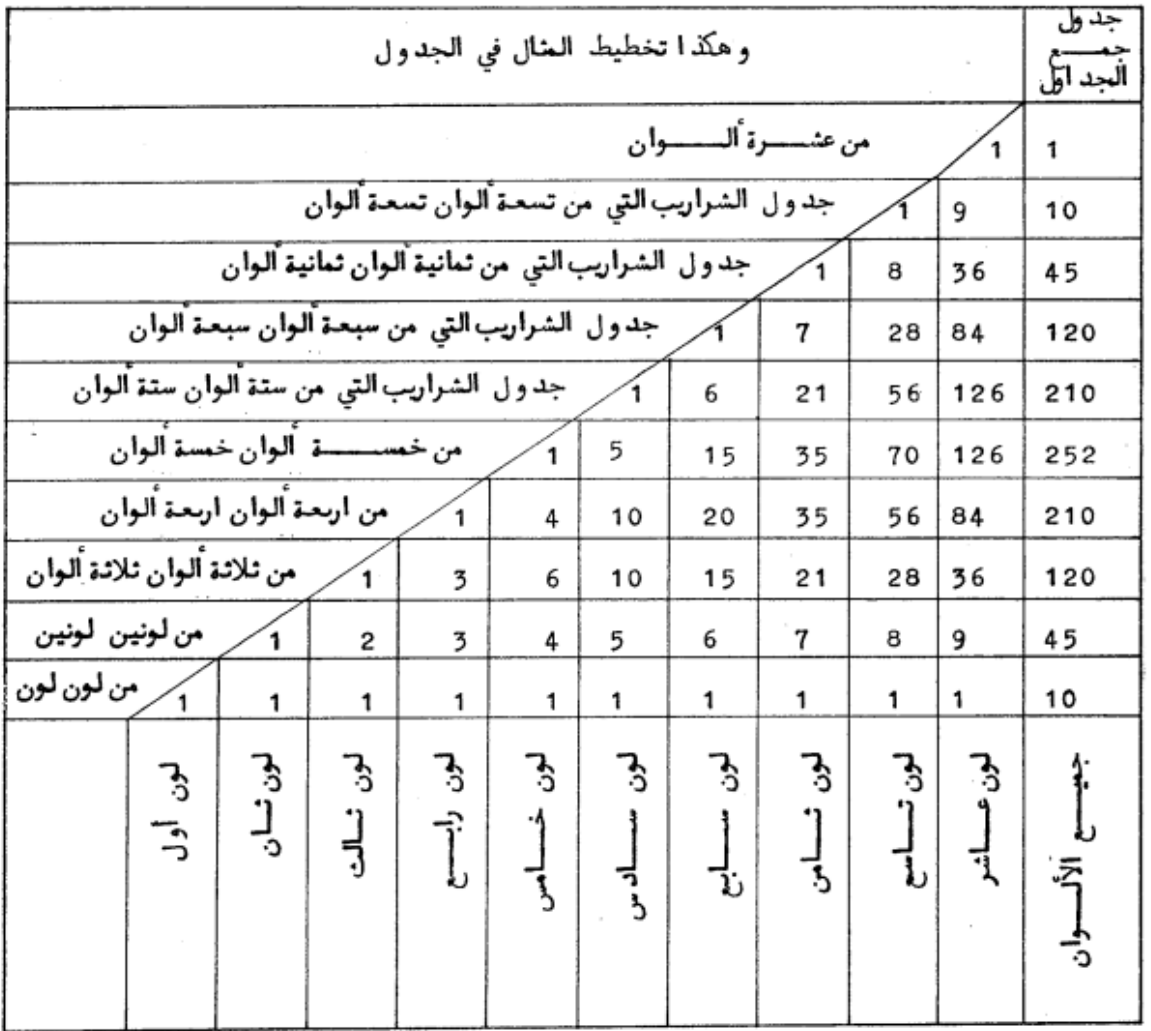

Fonte: Djebbar (1985, p. 101)

Na parte superior da figura 1 podemos ver as explicações de al-Muncim sobre as propriedades da tabela, as quais foram deixadas para a reflexão dos estudantes. Além disso, é possível observar o número 326 que representa a página do texto árabe original e, embora fora do escopo deste trabalho sobre notas de análise combinatória no mundo islâmico, representa um indício do histórico do documento.

Os outros problemas são relativos à quantidade de palavras que podem ser formadas a partir do alfabeto árabe que possui 28 letras. Por exemplo, o segundo e terceiro problemas dizem que

Problema II - Queremos encontrar um processo canônico para determinar o número de permutações de letras de uma palavra dado que o número de letras 
é conhecido e que nenhuma letra se repete ${ }^{6}$. (DJEBBAR, 1985, p.55, tradução nossa)

Problema III - Queremos conhecer o número de permutações das letras de uma palavra dado que o número de letras é conhecido e uma letra ou duas se repetem um determinado número de vezes? ${ }^{7}$ (DJEBBAR, 1985, p. 56. Tradução nossa).

Observe que o grau de dificuldade dos problemas vai aumentando e al-Muncim vai construindo sua teoria de análise combinatória passo a passo com os estudantes. $\mathrm{O}$ primeiro problema foi proposto de modo que fosse possível a manipulação concreta do raciocínio, o segundo já é teórico sem repetição de letras em uma permutação e o terceiro similar ao segundo, mas com possibilidades de repetição. Uma interpretação anacrônica do segundo problema é apresentada por Berggren (2016) que afirma que nele al-Muncim estabelece como se calcula o fatorial de um número.

No início do capítulo XI, al-Muncim esclarece que ele aprofunda o manuscrito de al-Khalil e que encontrou todas as possibilidades de combinações de palavras de uma a cinco letras que existem na Língua Árabe. Segundo ele:

\begin{abstract}
Al-Khalil - que Deus o seja misericordioso - indicou somente o número de configurações de palavras nas quais as letras não se repetem. Quanto as palavras que possuem letras que se repetem, assim como o número de palavras compostas por cinco ou seis letras do alfabeto ou então as letras são todas distintas ou uma, duas ou todas se repetem, assim como o número delas que esta seção se dedica ${ }^{8}$ (DJEBBAR, 1985, p. 49, tradução nossa).
\end{abstract}

É sobre o sábio indicado por al-Muncin que trataremos a próxima seção de nosso texto. Al-Khalil ibn Ahmad al Farahidi nasceu em Omã e viveu a maior parte de sua vida em Baçorá, onde atualmente se localiza o Iraque. Ele era linguista e lexicógrafo e estava interessado em construir um dicionário da língua árabe, o que é, segundo historiadores, o primeiro léxico do árabe.

\footnotetext{
${ }^{6}$ Nous voulons connaître un procédé canonique pour [déterminer le nombre] de permutations des lettres d'un mot dont le nombre de lettres est connu et dans lequel ne se rép ete aucune lettre.

${ }^{7}$ Nous voulons connaître le nombre de permutations des lettres d'un mot dont le nombre de lettres est connu et dont une lettre ou deux ou plus sont répétées un nombre connu de fois.

${ }^{8}$ Al-Khalil - que Dieu lui soit miséricordieux - a indiqué seulement le nombre de configurations du mot dans lequel ne se répètent pas de lettres. Quant aux mots dont les lettres se répètent, ainsi que le nombre de mots quintilitères ou sextilitères composés de lettres de l'alphabet et dont les lettres sont toutes distinctes ou dont une d'entre elles, ou deux, ou l'ensemble, sont répétées, ainsi que le dénombrement de tout cela, c'est cette sextion qui en renferme [l'étude].
} 


\section{Al-Khalil}

Como al-Muncim já anunciou, al-Khalil ibn Ahmad al Fararidi era linguista e lexicógrafo e desenvolveu um raciocínio de análise combinatória para palavras de até cinco letras. Ele é pouco conhecido entre os professores e historiadores da matemática, mas na Linguistica Árabe é bastante reconhecido sendo atribuída a ele a construção do primeiro dicionário da Língua Árabe. Esse interesse de linguista pela formalização da Língua Árabe, gramática e construção do léxico, é justificado por Rashed (1994) como, inicialmente, religioso. Havia a necessidade de criar um padrão para a língua árabe que é a língua escolhida por Alá para se transmitir a fé. Não entraremos neste trabalho em detalhes sobre a semântica e características do árabe, mas apresentaremos aqui algumas discussões para entendermos a necessidade da análise combinatória por linguistas e lexicógrafos.

Os primeiros léxicos eram constituídos de palavras que já estavam fora de uso ou com significados difíceis, como consequência disso o escopo dos léxicos era limitado e a ordem em que apareciam as palavras era meramente semântica (RASHED, 1994). Nesse sentido, al-Khalil foi o primeiro a construir um léxico diferente dos anteriores, ou seja, com todas as palavras da língua árabe e sua obra é conhecida pelo Kitab al- 'Ayn, O livro dos dois modos. Al-Khalil ibn Ahmad al-Fararibi nasceu no grande centro cultural de Omã, no Iraque no ano de 718 d.C. e morreu aos 70 anos de idade em Baçorá, também no Iraque.

Para a tarefa que ele se propôs de construir o léxico era necessário generalizar o modo semântico como as palavras eram apresentadas, o que não era uma tarefa muito clara e simples. Uma primeira solução era enumerar todas as palavras do idioma, o que é exaustivo, mas deve ser realizado por meio de uma bijeção entre as palavras e a semântica. Rashed (1994) ressalta que al-Khalil utilizou o conhecimento que ele tinha de música. Ao estudar as características morfológicas da Língua Árabe e Semítica, ele notou a importância das raízes na formação de novos vocábulos, sendo que o número de raízes não era grande. As raízes das palavras em árabe são limitadas a cinco letras, sendo a maioria de três letras. Por isso al-Khalil calcula as permutações de palavras de até cinco letras, como afirmou al-Muncim.

Al-Khalil deveria, portanto, realizar os cálculos do número de combinações sem repetição das letras do alfabeto sendo variadas de 2 a 5 consoantes e calcular o número 
de permutações de cada grupo. Rashed (1994) explica, anacronicamente por meio de símbolos atuais, que o que ele fazia era calcular que o arranjo de $\mathrm{n}$ elementos escolhidos p a p era o produto do fatorial de $n$ pela combinação de $n$ elementes escolhidos $\mathrm{p}$ a $\mathrm{p}$, ou seja, $A_{n, p}=n ! C_{n, p}$. Nessa perspectiva, para al-Khalil $n$ é o número de letras do alfabeto (no caso árabe igual a 28) e r variando de 2 a 5, que são as raízes. Rashed (1994) ainda apresenta uma citação de al-Khalil em uma nota de rodapé na qual ele justifica esse raciocínio e a justificativa de se calcular somente palavras de até cinco letras. Segundo ele:

\begin{abstract}
em árabe, nenhuma construção de substantivos ou verbos é composta por mais de cinco consoantes. Para quaisquer adjuntos que excedam cinco consoantes sejam encontrados em um substantivo ou verbo, saiba que eles são adjuntos a uma construção que não pertence à raiz da palavra9 ${ }^{9}$ (AL-KHALIL, 1967, p.55 apud RASHED, 1994, p.273, tradução nossa).
\end{abstract}

Não entramos em muitos detalhes de como al-Khalil calculava as permutações que precisava por depender de conhecimentos de Língua Árabe mais aprofundados, mas fica claro que ele utilizava um raciocínio combinatório de modo prático que foi usado por seus estudantes para terminar a construção do primeiro léxico de Língua Árabe. Darwish (1955) ressalta que no século XIII nenhuma língua europeia tinha um dicionário próprio apesar de existirem dicionários chineses. Entretanto, ele ressalta não haver evidências de que al-Khalil soubesse chinês ou tivesse tido acesso a tais trabalhos.

\title{
Considerações Finais
}

Trouxemos três trabalhos islâmicos com contribuições para a Análise combinatória que não foram contemporâneos, mas que tiveram seus conhecimentos transmitidos para gerações futuras. Foram notas históricas com informações de como a combinatória teve influências não só de matemáticos, mas de linguistas como foi visto em al-Khalil, no século VIII, cujo trabalho foi apropriado por al-Muncim, no século XIII. Este último aprofundou o caráter matemático do raciocínio e produziu conhecimentos para além do triângulo aritmético que já era conhecido pelos islâmicos, fazendo,

\footnotetext{
${ }^{9}$ In Arabic no construction of noun or verb is composed of more than five consonants. For any adjunctions exceeding five consonants to be found in a noun or verb, know that they are adjunctions to a construction which does not belong to the roor of the word.
} 
entretanto, comparações entre o raciocínio recorrente e as tabelas pelo fato de seu manuscrito ser dedicado a estudantes e apresentar um caráter mais didático.

Nesse sentido, enfatizamos que conhecer outras contribuições não eurocêntricas relativas à Análise combinatória pode ser útil aos professores em suas aulas. Oliveira, Rosa e Viana (2014, p.115) afirmam que:

\begin{abstract}
a História da Matemática pode servir como um instrumento para contextualizar as atividades curriculares matemáticas elaboradas pelos professores de acordo com os contextos social, econômico e cultural, nos quais ocorreu o desenvolvimento do conteúdo matemático. Possibilita-se, portanto, o entendimento do porquê da necessidade do estudo de determinados conteúdos matemáticos.
\end{abstract}

Essas notas históricas apresentam, portanto, alguns porquês da análise combinatória tanto em linguística com al-Khalil como na própria matemática com alBanna e al-Muncim. Por último, esperamos que o caráter não eurocêntrico que possa ter sido levantado nessas curtas informações leve ao conhecimento de professores da Educação Básica e pesquisadores em História da Matemática a contribuição islâmica também para a Análise combinatória. Desse modo, pesquisas mais profundas podem ser realizadas em Língua Portuguesa baseadas em originais traduzidos e em referências bibliográficas secundárias.

\title{
Referências
}

ABALLAGH, M. Raf ${ }^{\mathfrak{c}}$ al-Hijab d'Ibn al-Banna. Edition critique, Traduction, Etude philosophique et Analyse mathématique. 1988. 744f. Tese (Doutorado) - Universite de Paris I. Pantheon Sorbonne. Philosophie. Paris, 1988.

ABDELJAOUAD, M. Quelques éléments d'histoire de l'analyse combinatoire. Journées Nationales de l'ATSM, Mahdia: Tunísia. 2002.

BASTOS, T. A. Modelagem na Educação Matemática para o desenvolvimento de conceitos de Análise combinatória em uma escola particular no Vale do Rio Doce em Minas Gerais. 2020. 444 f. Dissertação (Mestrado) - Universidade Federal de Ouro Preto, Instituto de Ciências Exatas e Biológicas, Departamento de Educação Matemática, Programa de Pós-Graduação em Educação Matemática. Ouro Preto, 2019. Disponível em: https://www.repositorio.ufop.br/handle/123456789/11916. Acesso em: 28 nov. 2020 .

BERGGREN, John Len. Episodes in the Mathematics of Medieval Islam. Second Edition. Berlin, Heidelberg, New Your: Springer, 2016. 
DARWISH, A. Al-Khalil Ibn Ahmad and the Evolution of Arabic Lexicography. (Tese de Doutorado). University of London: School of Oriental and African Studies. 1955.

DJEBBAR, A. L'analyse combinatoire au Maghreb: l'exemple d'Ibn Muncim (XIIe - XIIIIe siècles), Paris, Université Paris-Sud. 1985.

MARTINS, M. C. S. BONFIM, S. H. Análise combinatória: um estudo via História da Matemática. Coleção História da Matemática para Professores. Natal: Sociedade Brasileira de História da Matemática, 2011.

MOREY, B; OLIVEIRA, D. P. A.; PEREIRA, A. P.; Tópicos de História da Matemática Islâmica Medieval. Coleção História da Matemática para Professores. Natal: Livraria da Física, 2021.

OLIVEIRA, D. P. A. ROSA, M. VIANA, M. C. V. A Perspectiva Sociocultural da História da Matemática na sala de aula: possibilidades e limites. REMATEC. Natal (RN). Ano 9. N. 16. maio-ag. P.107-129. 2014. Disponível em: http://www.rematec.net.br/index.php/rematec/issue/view/17 Acessado em: 15 nov. 2020.

PONTES, L. M. BATISTA, A. N. S. PEREIRA, A. C. C. A inserção de textos originais na disciplina de História da Matemática a partir de um problema do documento Sea Island Mathematical Manual. REVEMOP. Ouro Preto (MG). V. 3. p. 1-19. 2021. Disponível em:

https://periodicos.ufop.br:8082/pp/index.php/revemop/article/view/4671/3619 Acessado em: 20 jan. 2021.

RASHED, R. The Development of Arabic Mathematics: Between Arithmetic and Algebra. Tradução: ARMSTRONG, Angela. Boston Studies in the Philosophy of Science. V. 156. 1994.

VASQUEZ, C. M. R.; NOGUTI, F. C. H.. Análise combinatória: alguns aspectos históricos e uma abordagem pedagógica. In: VIII Encontro Nacional de Educação Matemática. 2004. Disponível em:

http://www.sbembrasil.org.br/files/viii/pdf/05/1MC17572744800.pdf . Acesso em: 10 out. 2020.

Recebido em: 26 / 02 / 2021

Aprovado em: 17 / 04 / 2021 